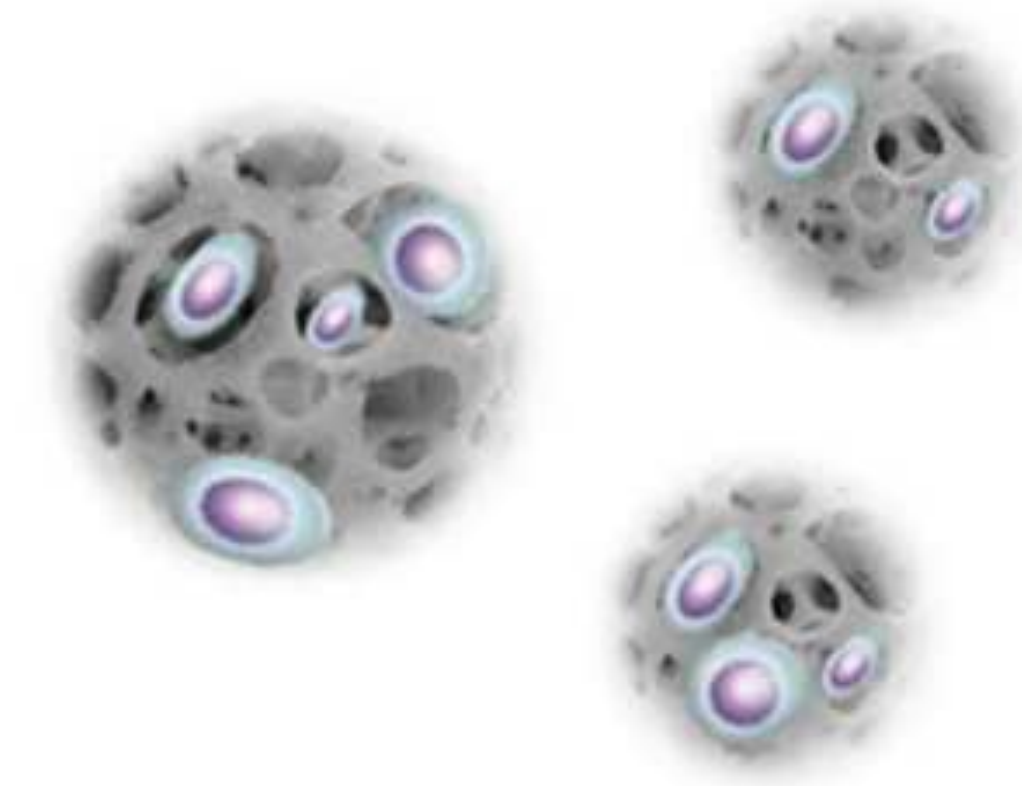

Human dental pulp stem cell/ Nanofibrous spongy microsphere aggregates
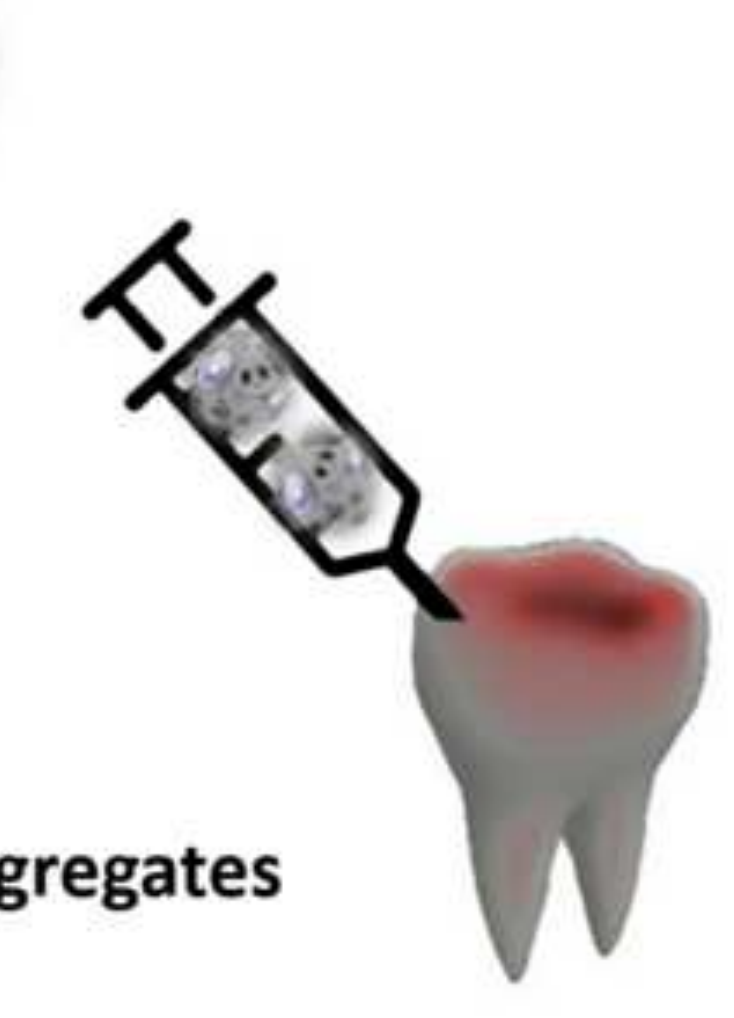


\section{Nanofibrous Spongy Microspheres for the Delivery of Hypoxia-primed Human Dental Pulp Stem Cells to Regenerate Vascularized Dental Pulp}

Rong Kuang a, b , Zhanpeng Zhang ${ }^{\mathrm{c}}$, Xiaobing Jin ${ }^{\mathrm{a}}$, Jiang $\mathrm{Hu}^{\mathrm{a}}$, Songtao Shi ${ }^{\mathrm{f}}$, Longxing $\mathrm{Ni}^{\mathrm{b},{ }^{* *}}$, Peter X. Ma ${ }^{\mathrm{a}, \mathrm{c}, \mathrm{d}, \mathrm{e}, *}$

${ }^{a}$ Department of Biologic and Materials Sciences, University of Michigan, Ann Arbor, MI 48109, USA.

${ }^{\mathrm{b}}$ State Key Laboratory of Military Stomatology, Department of Operative Dentistry and Endodontics, School of Stomatology, Fourth Military Medical University, Xi'an 710032, People's republic of China.

${ }^{\mathrm{c}}$ Department of Biomedical Engineering, University of Michigan, Ann Arbor, MI 48109, USA.

${ }^{\mathrm{d}}$ Macromolecular Science and Engineering Center, University of Michigan, Ann Arbor, MI 48109, USA.

${ }^{\mathrm{e}}$ Department of Materials Science and Engineering, University of Michigan, Ann Arbor, MI 48109, USA.

f Department of Anatomy and Cell Biology, School of Dental Medicine, University of Pennsylvania, 240 S. 40th Street, Philadelphia, PA 19104, USA

* Corresponding author. Department of Biologic and Materials Sciences, 1011 North University Ave., Room 2211, University of Michigan, Ann Arbor, MI 48109-1078, USA. Tel.: +1 734764 2209; fax: +1 734647 2110; email address: mapx@ umich.edu

${ }^{* *}$ Corresponding author. Tel.: +86 29 84776476; fax: +86 29 83224432; E-mail addresses: nilx2007@gmail.com 


\begin{abstract}
Dental pulp infection and necrosis are widespread diseases. Conventional endodontic treatments result in a devitalized and weakened tooth. In this work, we synthesized novel star-shaped polymer to self-assemble into unique nanofibrous spongy microspheres (NFSMS), which were used to carry human dental pulp stem cells (hDPSCs) into the pulp cavity to regenerate living dental pulp tissues. It was found that NF-SMS significantly enhanced hDPSCs attachment, proliferation, odontogenic differentiation and angiogenesis, as compared to control cell carriers. Additionally, NF-SMS promoted vascular endothelial growth factor (VEGF) expression of hDPSCs in a 3D hypoxic culture. Hypoxia-primed hDPSCs/NF-SMS complexes were injected into the cleaned pulp cavities of rabbit molars for subcutaneous implantation in mice. After 4 weeks, the hypoxia group significantly enhanced angiogenesis inside the pulp chamber and promoted the formation of ondontoblast-like cells lining along the dentin-pulp interface, as compared to the control groups (hDPSCs alone group, NF-SMS alone group, and hDPSCs/NF-SMS group pre-cultured under normoxic conditions). Furthermore, in an in situ dental pulp repair model in rats, hypoxia-primed hDPSCs/NF-SMS were injected to fully fill the pulp cavity and regenerate pulp-like tissues with a rich vasculature and a histological structure similar to the native pulp.
\end{abstract}

Key words: Regeneration, stem cell, dental pulp, hypoxic, biomaterial, microcarrier. 


\section{Introduction}

The vitality of the entire tooth is supported by the dental pulp, which is responsible for nutritional supply, dentin production, and tooth sensation [1]. However, dental pulp is vulnerable to infections resulted from mechanical, chemical, thermal or microbial irritants. In addition to unbearable pain, dental pulp infection can lead to irreversible pulp necrosis and interruption of dentin formation, which can result in unclosed apical foraman in young permanent teeth or the formation of large pulp chambers [2]. The current endodontic treatment of irreversible pulp disease, known as root canal treatment (RCT), cannot restore the function of dental pulp and thereby results in a permanently devitalized tooth more susceptible to structural failure and re-infection due to coronal leakages or microleakages [3]. Restoring the functions of dental pulp through tissue regeneration may potentially resolve these issues [4-6]. However, a key challenge to functional dental pulp regeneration is to rebuild the complex, highly organized histological structure of the native pulp, which is vascularized and contains several types of cells in different zones (e.g. microvasculature in the central region and odontoblasts in the peripheral lining against the dentinal wall).

Human dental pulp stem cells (hDPSCs) are an excellent cell source for dental pulp regeneration, because hDPSCs are capable of odontogenic, neurogenic and angiogenic differentiations $[7,8]$ to form the key cell types in natural dental pulp. In addition, hDPSCs are advantageous for clinical application because of their easy isolation [7] from third molars, extracted or broken teeth [9-11], and their regenerative capacity after cryopreservation [12]. To fully harness the regenerative potential of the hDPSCs, an advanced cell carrier is needed to effectively induce angiogenesis and direct hDPSCs 
differentiation to regenerate the entire vascularized dental pulp [7, 13-16]. To design cell carriers for dental pulp regeneration, structural features at multiple scales have to be considered. At the nano-scale, nano-fibrous (NF) structure can mimic the fibrous structure of extracellular matrix (ECM) and promote hDPSCs attachment, proliferation, and odontogenic differentiation $[17,18]$. At the micro-scale, a porous structure with interconnected pores is required for efficient cell seeding/ingrowth, mass transfer, and vasculature formation [19-21]. At the macro-scale, cell carriers should be injectable to enable cell delivery into the small, irregularly-shaped dental cavity using a minimal invasive procedure $[22,23]$.

In addition to physical cues, angiogenic factors are required to induce and promote blood vessel formation, which is critical to tissue vitality and pulp histological organization [2427]. Resembling the hypoxic condition in dental pulp cavity [28], culturing cells under a low oxygen tension in vitro activates transcriptional factor HIF-1 $[29,30]$ and increases vascular endothelial growth factor (VEGF) expression [31-34]. HIF-1 $\alpha$ binds to the VEGF gene promoter and recruits other transcriptional regulators, thereby enhancing VEGF gene expression [35]. However, thus far, such studies were conducted in 2D culture plates [36]. Little is known about the hypoxia effect on hDPSCs in 3D culture, especially on microspheres.

In this work, a series of star-shaped block copolymers was synthesized for the first time and was fabricated into novel nanofibrous spongy microspheres (NF-SMS), which integrated the desired synthetic NF architecture and interconnected micro-sized pores into injectable microspheres. We then investigated the effect of hypoxic treatment on hDPSCs seeded on the newly developed NF-SMS in a suspension culture, and examined their 
hypoxia-induced VEGF gene expression. Furthermore, we evaluated the injectable, hypoxia-primed hDPSCs/NF-SMS complexes for dental pulp regeneration in both a subcutaneous tooth implantation model and an in situ pulp regeneration model.

\section{Methods}

Synthesis of SS-PLLA-b-PLYS

Star-shaped poly(L-lactic acid)-block-poly(L-lysine) (SS-PLLA-b-PLYS) was prepared (supplemental data, Fig.S1). Briefly, poly(amidoamine) dendrimer with 16 surface hydroxyl groups (PAMAM-OH, generation 2, Sigma-Aldrich) was used to initiate the ring opening polymerization of monomer L-lactide (Sigma-Aldrich) under vacuum at $120-130^{\circ} \mathrm{C}$ to synthesize star-shaped poly(L-lactic acid) (SS-PLLA). The hydroxyl end groups of SS-PLLA were then converted into amino groups to form SS-PLLA-NH ${ }_{2}$. SSPLLA- $\mathrm{NH}_{2}$ was used to initiate a second ring-opening polymerization of lysine $\mathrm{N}$ carboxyanhydride with a carbobenzyloxy protecting group (Z-LYSNCA). SS-PLLA-NH $\mathrm{N}_{2}$ and Z-LYSNCA were dissolved in dry dimethylformamide and the solution was stirred at $30^{\circ} \mathrm{C}$ for 72 hours with nitrogen purged throughout the reaction. The product SS-PLLAb-PLYS(Z) was then dissolved in trifluoroacetic acid and treated with hydrogen bromide/acetic acid (volume ratio $=1: 3$ ) solution under nitrogen at $0^{\circ} \mathrm{C}$ for 1 hour to remove the protecting group to obtain SS-PLLA-b-PLYS. Detailed characterization of the materials can be found elsewhere [37].

Preparation of Nanofibrous Spongy Microspheres (NF-SMS)

NF-SMS were produced from SS-PLLA-b-PLYS through a "reversed" emulsification process. Briefly, the polymer was dissolved in THF at $50{ }^{\circ} \mathrm{C}$ with a concentration of $2.0 \%$ 
$(\mathrm{wt} / \mathrm{v})$. The polymer solution was quickly added into glycerol $\left(50^{\circ} \mathrm{C}\right)$ under rigorous mechanical stirring (speed 7, MAXIMA, Fisher Scientific Inc.). Five minutes later, the mixture was quickly poured into liquid nitrogen. After 10 minutes, ice/water mixture was added to exchange solvent for 24 hours. The spheres were then sieved and washed with distilled water five times to remove glycerol residue. The spheres were then lyophilized for 2 days.

Preparation of Nanofibrous Microspheres (NF-MS)

NF-MS were prepared following a previously described emulsification procedure [22]. PLLA was dissolved in $\mathrm{THF}$ at $50{ }^{\circ} \mathrm{C}$ with a concentration of $2.0 \%(\mathrm{wt} / \mathrm{v})$. Under rigorous mechanical stirring (speed 7, MAXIMA, Fisher Scientific Inc.), glycerol $\left(50^{\circ} \mathrm{C}\right)$ was slowly added into the polymer solution, and the stirring continued for 5 minutes. The mixture was then quickly poured into liquid nitrogen. After 10 minutes, ice/water mixture was added to exchange solvent for 24 hours. The spheres were then sieved and washed with distilled water five times to remove glycerol residue. The spheres were then lyophilized for 2 days.

\section{Preparation of Solid Microspheres (S-MS)}

Smooth (solid) microspheres were prepared using a conventional solvent evaporation method. The PLLA was dissolved in dichloromethane at a concentration of $2 \%(\mathrm{wt} / \mathrm{v})$, and added into an aqueous poly(vinyl alcohol) (PVA, $\mathrm{M}_{\mathrm{w}}=89,000 \sim 98,000$, SigmaAldrich) solution $(2 \% \mathrm{wt} / \mathrm{v})$. The mixture was then subjected to rigorous stirring (speed 7 , MAXIMA, Fisher Scientific Inc.) for 24 hours. The spheres were then sieved and washed with distill water five times to remove PVA residue. The spheres were then lyophilized for 2 days. 
Scanning electron microscopy (SEM) observation

The morphology of the polymer microspheres was examined using SEM (Philips XL30 FEG) with an accelerating voltage of $10 \mathrm{kV}$. The samples were coated with gold using a sputter coater (DeskII, Denton vacuum Inc) with a gas pressure of 50 mtorr and a current of $40 \mathrm{~mA}$.

\section{Set- up of hypoxia bioreactor culture system}

We set up a hypoxia-bioreactor for the hypoxic culture of hDPSCs on microspheres. The system was composed of a gas supply, a spinner flask and a container. After placing the spinner flask in the container, the system was vacuumed and purged with a gas mixture containing $2 \% \mathrm{O}_{2}\left(5 \% \mathrm{CO}_{2}\right.$ and $\left.93 \% \mathrm{~N}_{2}\right)$ for three times to create a hypoxic environment. The container was then sealed and placed on a magnetic stirring plate, and the entire system was placed in a $37{ }^{\circ} \mathrm{C}$ incubator. This self-made hypoxia-bioreactor was able to generate either hypoxic or normoxic environment by using a desired gas mixture.

\section{Cell culture and seeding on microspheres}

Human DPSCs were a gift from the Center of Craniofacial Molecular Biology, School of Dentistry, University of Southern California, which were isolated according to a previously reported method [7]. The thawed hDPSCs were cultured in $\alpha$-modified essential medium ( $\alpha$-MEM) (Invitrogen, Carlsbad, CA) supplemented with $10 \%$ fetal bovine serum (FBS) (Invitrogen) and 1\% Penicillin- Streptomycin (Invitrogen) in a humidified incubator at $37^{\circ} \mathrm{C}$ with $5 \% \mathrm{CO}_{2}$. The medium was changed every two days and hDPSCs of passages 3- 6 were used in the subsequent studies.

The microspheres were soaked in $70 \%$ ethanol to pre-wet them for 30 minutes and then the ethanol was exchanged with phosphate-buffered saline (PBS, GIBCO) three times (30 
minutes each). The microspheres were then washed with $\alpha$-MEM containing $10 \%$ FBS for 30 minutes. $8 \times 10^{6}$ cells and $5 \times 10^{5}$ microspheres were mixed in $2 \mathrm{ml}$ culture medium in a $15 \mathrm{ml}$ centrifuge tube on an orbital shaker at $12 \mathrm{rpm}$ for 4 hours. Then the mixture was transferred into a spinner flask (Wheaton Industries Inc., Millville, NJ) and maintained in $80 \mathrm{ml}$ culture medium. The stirring speed was maintained at $80 \mathrm{rpm}$.

\section{Observation of $h D P S C s / m i c r o s p h e r e s$}

The hDPSCs on NF-SMS under different culture conditions were rinsed in PBS once, fixed in $2.5 \%$ glutaraldehyde, and post-fixed in $1 \%$ osmiumtetroxide for 1 hour. Samples were dehydrated in a series of ethanol solution with increasing concentrations, and then in hexamethyldisilizane. The samples were then sputter-coated with gold using a sputter coater (DeskII, Denton vacuum Inc) and observed under an SEM (Philips XL30 FEG) at $10 \mathrm{kV}$.

DNA quantification assay

To examine the proliferation of hDPSCs on microspheres under different culture conditions, the cell-sphere complexes were rinsed with PBS and homogenized in 1 xDNA assay buffer and lysis buffer (Sigma), which were then incubated at $37^{\circ} \mathrm{C}$ for 1 hour.

Cell lysis was centrifuged at 5,000 $\mathrm{g}$ at room temperature for 3 minutes. The supernatant was collected for DNA content determination using fluorescence assay with Hoechst 33258 dye (Sigma) [38].

\section{Real time PCR}

After hypoxia or normoxia treatment for 1, 3, 7, 10 days, the total RNA of each sample was extracted using RNA Mini kit (Qiagen, Valencia, CA), with the first-strand cDNA reversely transcribed using TaqMan reverse transcription reagents (Applied Biosystems, 
Foster City, CA). Real-time PCR quantification of VEGF mRNA was performed in a 7500 Real-Time PCR System (Applied Biosystems) using a Taqman probe (Hs00900055_m1).

\section{Immunofluorescence Staining}

The hDPSCs/NF-SMS complexes were taken out from the spinning flask, washed using PBS, fixed by $4 \%$ formaldehyde solution, and treated with $0.1 \%$ triton for 3 minutes. The samples were then blocked with PBS containing $2 \%$ bovine serum albumin at room temperature for 20 minutes. The cells were then incubated with primary antibodies of DSPP (Santa Cruz Biotechnology Inc, Santa Cruz, CA), human VEGF165b (R\&D Systems, Inc, Minneapolis, MN) or HIF-1 $\alpha$ (c-19) (Santa Cruz Biotechnology Inc) at $4^{\circ} \mathrm{C}$ overnight. The samples were then washed with PBS and subsequently incubated with fluorescein-conjugated secondary donkey anti-mouse IgG-FITC antibody (Santa Cruz Biotechnology Inc.) at room temperature in the dark for 45 minutes. The samples were then washed with PBS and incubated with Alexa Fluor 555 phalloidin (Life technologies Inc) for 30 minutes, followed by washing with PBS for 3 times. The samples were transferred into 8-well chambers and mounted with Vectashield mounting medium with DAPI (Vector Laboratories, Inc., Burlingame, CA). The samples were analyzed using a confocal microscope (Nikon TS-100, Tokyo, Japan).

\section{Subcutaneous injection model}

The animal surgical procedure was approved by the University Committee on Use and Care of Animals (UCUCA) at the University of Michigan. Nude mice (nu/nu) with an age range of 6-8 weeks (Charles River Laboratories, Wilmington, MA) were used in this study. Surgery was performed under general inhalation anesthesia with $2 \%$ isofluorane. 
The cell-sphere complexes cultured in hypoxic bioreactor for 3 days were the experimental group, and those pre-cultured under normoxic condition were the control group. Samples of each group were subcutaneously injected into nude mice in a random order $(n=6)$. Each mouse received 2 injections. Animals were sacrificed 4 weeks postinjection and samples were retrieved. Harvested specimens were immediately fixed in $10 \%$ formalin for 24 hours, which were then processed for histological examination using hematoxylin-eosin ( $\mathrm{H} \& \mathrm{E})$ and CD31 staining. The stained sections were observed under a microscope, and 3 fields of each injection in the two groups were chosen randomly under microscope and the neo blood vessels were counted.

\section{Subcutaneous tooth implantation model}

Sixteen molars with opened apical foraman were collected from 2-week-old rabbits. Dental pulps of the molars were completely removed. The empty teeth were rinsed with $70 \%$ ethanol once and PBS twice. DPSCs/NF-SMS complexes pre-cultured under hypoxic or normoxic condition for 3 days in bioreactors were injected into the rabbit molar pulp cavities, which have a volume of about $0.15 \mathrm{~cm}^{3}$ and a diameter of the apex opening of about $1.5-2 \mathrm{~mm}$. The rabbit pulp cavity and apex opening are similar to those of a human tooth. Nude mice (nu/nu) with an age range of 6-8 weeks (Charles River Laboratories) were used in this study. Surgery was performed on mice under general inhalation anesthesia with $2 \%$ isofluorane. Two midsagittal incisions were made on the dorsa and four subcutaneous pockets were created using blunt dissection. Four groups of the rabbit molars, filled with NF-SMS alone, hDPSCs alone, and with hypoxia or normoxia primed hDPSCs/NF-SMS complexes in the emptied pulp chambers were 
implanted into the subcutaneous pockets $(n=4)$, and the incisions were closed with staples. After 4 weeks of implantation, samples were harvested.

\section{In situ pulp regeneration model}

Nude rats (Charles River labs) with an age range of 6-8 weeks were used in this study $(n=6)$. Surgery was performed on rats under general inhalation anesthesia with $2 \%$ isofluorane. The endodontic treatment was as follows: the mouth of the animal was opened; the maxillary first molar was drilled using a thin bur (SS White Burs Inc., Lakewood Township, NJ); the entire pulp was removed and root canals were shaped using K-files (\#15- \#25) by hands. Root canals were irrigated using PBS and 5.25\% $\mathrm{NaClO}$ alternatingly and dried using absorbent points (Densply Maillefer, Tulsa, OK). hDPSCs/NF-SMS complexes primed under hypoxic conditions were injected into the prepared root canals, while those primed under normoxic conditions were injected into contralacteral first molar root canals. Instead of using composite resin for sealing, which requires good saliva isolation, an NF PLLA scaffold and a Fuji IX GP Glass ionomer (GC American Inc., Alsip, IL) were used to seal the entrance of the cavity in the rat mouth where saliva isolation was difficult to achieve. The mandibular first molar cusps were removed for occlusal reduction to protect the experimental teeth. Four weeks after the surgical procedure, the maxillary samples were harvested. For comparison, maxillary first molars received no treatment after pulp removal and contralateral healthy teeth were also collected as controls $(n=3)$.

\section{Histological analysis}

Harvested specimens were immediately fixed in $10 \%$ formalin for 24 hours, and tooth samples were decalcified in $10 \%$ ethylene diamine tetraacetic acid for 4 weeks prior to 
histological examination using H\&E staining. CD31 (Abcam, Cambrige, MA), VEGFa (Santa Cruz Biotechnology Inc) and DSPP (Santa Cruz Biotechnology Inc) primary antibodies were used for immunohistological staining.

Quantification of neo blood vessels

The CD31 immunohistologically stained sections were observed under a microscope, and several images were combined together to show the whole pulp cavity. The neo blood vessels were counted for each group $(n=3)$.

Statistical analysis

Numerical data were reported as mean \pm S.D. The experiments were performed twice to ensure reproducibility. To test the significance of observed differences between the study groups, Student's t-test was used. A value of $\mathrm{p}<0.05$ was considered to be statistically significant.

\section{Results}

NF-SMS providing a beneficial microenvironment for hDPSCs attachment, proliferation and angiogenesis

Star-shaped poly(L-lactic acid)-block-poly(L-lysine) (SS-PLLA-b-PLYS) was synthesized and assembled into nanofibours spongy microspheres (NF-SMS). At the micro-scale, NF-SMS had an interconnected pore structure throughout the entire microsphere (Fig.1a Left). The pores in the NF-SMS had an average diameter of approximately $15 \mu \mathrm{m}$, which were highly interconnected (the connecting openings were about $10 \mu \mathrm{m}$ in size). At the nano-scale, NF-SMS were composed entirely of ECMmimicking nanofibers (Fig.1a Left). Due to the co-presentation of biomimetic nanofibers and a highly porous structure, NF-SMS were hypothesized to simultaneously promote 
DPSC seeding, proliferation, and vascularized dental pulp regeneration. Nanofibrous microspheres without a porous structure and conventional smooth microspheres without the NF structure were used as controls (Fig.1a Middle, Right) to test this hypothesis. After $24 \mathrm{hr}$ of cell seeding, hDPSCs attached to both the outer surfaces and the interior pores of the NF-SMS with abundant cellular processes (Fig.1b Left). In comparison, hDPSCs only attached to the outer surface of the nanofibrous microspheres and exhibited fewer cellular processes (Fig.1b Middle). In the smooth microspheres group, the cells attached on the outer surfaces of the microspheres with a flat morphology and fewer cellular processes (Fig.1b Right). The DNA quantification revealed that the hDPSCs proliferated markedly faster on the NF-SMS than on the nanofibrous microspheres or smooth microspheres during the first 12 days of in vitro culture (Fig.2a), likely benefitted from the ECM-mimicking NF architecture and the porous structure. After subcutaneous injection of cell/microsphere complexes into mice for 4 weeks, hematoxylin and eosin (H\&E) staining of the harvested samples revealed more tissue and blood vessel formation in the NF-SMS group than in the nanofibrous microspheres group or smooth microspheres group (Fig.2b), where the highly porous structure and fast degradation rate of the NF-SMS likely facilitated the neo vasculature formation. Most of the NF-SMS degraded 4 weeks after injection, whereas the majority of the smooth microspheres and a large number of nanofibrous microspheres still persisted in the neo tissue (Fig.2b). Detailed degradation assessment of the materials can be found in the literature [22]. These results supported our hypothesis, indicating that NF-SMS provided a beneficial microenvironment for hDPSCs distribution, proliferation, and angiogenesis. Therefore, NF-SMS were selected for further studies. 
Hypoxia culture of DPSCs on NF-SMS enhanced angiogenesis

Effective hypoxia priming of hDPSCs with NF-SMS was established in a 3D suspension culture system. Specifically, the suspension of the hDPSCs/NF-SMS was subject to a gas mixture of $2 \% \mathrm{O}_{2}, 5 \% \mathrm{CO}_{2}$ and $93 \% \mathrm{~N}_{2}$ under gentle stirring in a bioreactor maintained at $37{ }^{\circ} \mathrm{C}$ (Fig.3a). Normoxic culture of the hDPSCs/NF-SMS in a gas mixture of $21 \% \mathrm{O}_{2}$, $5 \% \mathrm{CO}_{2}$ and $74 \% \mathrm{~N}_{2}$ served as the control group. To examine the hypoxia effect, we performed immunofluorescence staining for HIF-1 $\alpha$ [29, 30]. Positive immunofluorescence staining of HIF-1 $\alpha$ was observed in the hypoxia group at all tested time points $(1,3,7$ and 10 days), whereas the staining was negative in the normoxia group (Fig.3b).

Immunofluorescence staining showed that hDPSCs started to express VEGF at day 3 (Fig.3c), implicating that VEGF expression occurred as a result of the earlier HIF-1 $\alpha$ activation (detected at day 1). Real-time PCR analysis showed that the VEGF was maximally up-regulated at day 3 and remained highly up-regulated at day 7 in hypoxic culture when compared to normoxic culture (Fig.4b). Therefore, hypoxic culture was carried out for 3 days in vitro to maximize the angiogenic potential of hDPSCs for the following in vivo studies. In a subcutaneous injection model in mice, pulp-like tissue formed both in the hypoxia group (pre-cultured under hypoxia) and normoxia group (precultured under normoxia) 4 weeks after the injection, but a much higher level of angiogenesis was observed in the hypoxia group (Fig.5a), which was quantified by counting the neo blood vessels (Fig.5b). Taken together, hypoxia-priming of hDPSCs on NF-SMS induces VEGF expression and enhances the subsequent angiogenesis in vivo. Pulp tissue formation in ectopically implanted tooth 
In a subcutaneous tooth implantation model, hypoxia-primed hDPSCs/NF-SMS complexes were injected into the cleaned pulp cavities of rabbit molars, and the whole constructs were implanted into nude mice subcutaneously. Three control groups were investigated: a normoxia-treated hDPSCs/NF-SMS group, a NF-SMS group (without cells), and a hDPSCs group (without a carrier). Upon tissue harvest at 4 weeks, the implanted molars appeared to have larger red areas in the hypoxia group than those in the other three groups (Fig.6a), indicating more blood vessels formed in the hypoxia group than in the other three groups. CD31 staining of the endothelial cells confirmed rich micro-vessel formation in the hypoxia group, as compared to the other three groups (Fig.6b\&c). H\&E staining showed a thick layer of cells lining along the pulp-dentin interface in the hypoxia group (Fig.6c), which were identified to be odontoblast-like cells with positive dentin sialophosphoprotein (DSPP) staining along the dentin wall. This histological structure resembled the natural dental pulp-dentin interface. In contrast, the normoxia group showed a positive DSPP staining evenly distributed throughout the whole reparative tissue, and the injection of hDPSCs alone resulted in negative DSPP staining (Fig.6c).

\section{In situ pulp-like tissue regeneration}

With encouraging results in regenerating pulp tissues in ectopically implanted tooth model, hypoxia-primed hDPSCs/NF-SMS were evaluated in an in situ pulp regeneration model in nude rats. The pulp tissues in root canals were removed before the hypoxia- or normoxia-primed hDPSCs/NF-SMS were injected. After 4 weeks, samples were harvested for evaluation. 
H\&E staining confirmed that hDPSCs/NF-SMS could fully fill the molar canals through injection and promoted pulp-like tissue formation with intimate integration with the native dentin (Fig.7). Most of the NF-SMS degraded after 4 weeks. The hypoxia-primed hDPSCs/NF-SMS regenerated pulp-like tissues with a much higher vascularity than the normoxia group (Fig.7). When the empty pulp cavity was un-treated, only a small amount of connective tissue was formed, with neither blood vessel formation nor a pulplike structure (Fig.7). Consistent with the subcutaneous tooth implantation model, IHC staining showed a positive DSPP staining along the dentin-pulp interface in the hypoxia group, but a weaker DSPP staining in the normoxia group. In addition, the cells in the hypoxia-primed group showed the columnar arrangement at the dentin-pulp interface, which is similar to the odontoblasts along the dentin-pulp interface in the native teeth. Collectively, the above data demonstrated that hypoxia-primed hDPSCs/NF-SMS effectively regenerated a vascularized dental pulp in an in situ pulp regeneration model.

\section{Discussion}

Dental pulp tissue engineering aims to develop effective approaches to restoring the structure and function of diseased dental pulp [4]. However, this task is challenging because of the complex anatomical and histological structure of the dental pulp. First, the small, irregularly-shaped pulp cavity posts a significant challenge in scaffold design. Second, vascularity is critical to the vitality of the regenerated pulp tissue, but the pulp cavity has only one small apical foramen to allow angiogenesis. Third, dental pulp has a complex histological structure consisting of different types of cells in different zones, such as odontoblasts located peripherally to the pulp tissue. Novel approaches are therefore required to overcome these daunting challenges facing pulp tissue regeneration. 
Human DPSCs possess odontogenic, neurogenic and angiogenic differentiation capabilities $[7,8]$ to rebuild pulp tissues consisting of a mixture of cell types. Through innovative polymer synthesis and self-assembly at both the nano- and micro-scales, we developed unique NF-SMS as an advanced injectable cell carrier for hDPSCs in this work. The biomimetic nanofibers and porous structure of NF-SMS were hypothesized to simultaneously promote hDPSCs proliferation, differentiation and angiogenesis. Supporting this hypothesis, NF-SMS enhanced hDPSCs attachment (Fig.1b) and proliferation (Fig.2a) in vitro, and promoted more tissue formation with a richer vasculature in a subcutaneous injection model in mice (Fig.2b).

Biochemical stimuli are required to initiate angiogenesis, which is critical to the vitality and organization of the reparative tissue [25-27]. The vascular endothelial growth factor (VEGF) is a potent endothelial cell mitogen in vascularization and angiogenesis [39, 40], and has recently been studied as a morphogen in pulp tissue engineering. However, exogenous VEGF has a short half-life in vivo, is expensive, and requires a complicated delivery system. In this work, we demonstrated that culturing 3D hDPSCs/NF-SMS suspension under hypoxic conditions was capable of activating the HIF-1 $\alpha$ in hDPSCs (Fig.3b), and up-regulating VEGF expression (Fig.3c). Notably, when conventional smooth microspheres were used as the hDPSC carrier in a suspension culture, no hypoxia-induced VEGF expression was detectable (Fig.4a). It was reported that hypoxia activation and the associated signaling pathways were strongly stimulated by cell-cell interactions [41]. Therefore, possibly due to limited cell-cell interactions, hypoxia treatment failed to induce VEGF expression of hDPSCs seeded on smooth microspheres. Different from smooth microspheres, NF-SMS facilitated hDPSCs attachment three- 
dimensionally in the interconnected pores, thereby facilitating cell-cell interactions in 3D. As a result, hypoxia-induced VEGF expression was significantly up-regulated when NFSMS were used as the hDPSC carrier in a suspension culture.

With the advantages of the NF-SMS and the hypoxia treatment, the injectable, hypoxiaprimed hDPSCs/NF-SMS complexes were able to regenerate vascularized pulp-like tissues in both a subcutaneously tooth implantation model in mice and more importantly in a clinically relevant in situ dental pulp repair model in rats. In both models, hDPSCs/NF-SMS complexes could be injected to fill the entire pulp cavity and root canal, regenerating dental pulp-like tissues integrated to the dentinal wall. The hypoxiaprimed hDPSCs/NF-SMS group resulted in a higher vascularity and ondontoblast-like cells lining along the dentin-pulp interface, which resembled the histological structure of natural dental pulp. The high vascularity in the reparative tissue in the hypoxia group was likely to have regulated the tissue organization during regeneration [27]. A critical feature of the natural dentin-dental pulp interface is the lining of odontoblasts at the interface. The regeneration of the pulp-like tissue should resemble this structure, as indicated by the positive IHC staining at the interface instead of the whole pulp. This was observed in normal tissue and the hypoxia experimental group, where short columnar cells were stained brown along the dentin-pulp interface. It should be noted that there is no specific marker of odontoblasts to date, but we employed DSPP IHC staining and visual analysis of cell organization to determine that the cells along the dentin-pulp interface were odontoblast-like cells.

In conclusion, with the simplicity, minimal invasiveness and regenerative efficacy, hypoxia-primed hDPSCs/NF-SMS complexes have shown high potential as a novel next- 
generation therapy for dental pulp diseases. NF-SMS could also be used to regenerate other highly vascularized tissues using a minimally invasive procedure, especially those with a hard to reach irregular shape.

\section{Supporting Information}

Supporting Information is available.

\section{Acknowledgements}

The authors gratefully acknowledge financial support from the NIH (NIDCR DE022327, DE015384, and NHLBI HL114038: PXM), DOD (W81XWH-12-2-0008: PXM), and NSF (DMR-1206575: PXM).

\section{References}

[1] Huang GT. Dental pulp and dentin tissue engineering and regeneration: advancement and challenge. Front Biosci (Elite Ed) 2011;3:788-800.

[2] Cvek M. Prognosis of luxated non-vital maxillary incisors treated with calcium hydroxide and filled with gutta-percha. A retrospective clinical study. Endod Dent Traumatol 1992;8:45-55.

[3] Dammaschke T, Steven D, Kaup M, Ott KH. Long-term survival of root-canal-treated teeth: a retrospective study over 10 years. J Endod 2003;29:638-43.

[4] Hench LL, Polak JM. Third-Generation Biomedical Materials. Science 2002;295:1014-7.

[5] Nakashima M, Reddi AH. The application of bone morphogenetic proteins to dental tissue engineering. Nature Biotechnology 2003;21:1025-32.

[6] Miura M, Gronthos S, Zhao M, Lu B, Fisher LW, Robey PG, et al. SHED: Stem cells from human exfoliated deciduous teeth. Proceedings of the National Academy of Sciences 2003;100:5807-12.

[7] Gronthos S, Mankani M, Brahim J, Robey PG, Shi S. Postnatal human dental pulp stem cells (DPSCs) in vitro and in vivo. Proc Natl Acad Sci U S A 2000;97:13625-30.

[8] Nakashima M, lohara K, Sugiyama M. Human dental pulp stem cells with highly angiogenic and neurogenic potential for possible use in pulp regeneration. Cytokine Growth Factor Rev 2009;20:435-40.

[9] Atari M, Gil-Recio C, Fabregat M, Garcia-Fernandez D, Barajas M, Carrasco MA, et al. Dental pulp of the third molar: a new source of pluripotent-like stem cells. J Cell Sci 2012;125:3343-56.

[10] Ma D, Gao J, Yue J, Yan W, Fang F, Wu B. Changes in proliferation and osteogenic differentiation of stem cells from deep caries in vitro. J Endod 2012;38:796-802.

[11] Huang AH, Chen YK, Chan AW, Shieh TY, Lin LM. Isolation and characterization of human dental pulp stem/stromal cells from nonextracted crown-fractured teeth requiring root canal therapy. J Endod 2009;35:673-81.

[12] Perry BC, Zhou D, Wu X, Yang FC, Byers MA, Chu TM, et al. Collection, cryopreservation, and characterization of human dental pulp-derived mesenchymal stem cells for banking and clinical use. Tissue Eng Part C Methods 2008;14:149-56.

[13] Langer R, Vacanti JP. Tissue engineering. Science 1993;260:920-6.

[14] El-Backly RM, Massoud AG, El-Badry AM, Sherif RA, Marei MK. Regeneration of dentine/pulp-like tissue using a dental pulp stem cell/poly(lactic-co-glycolic) acid scaffold construct in New Zealand white rabbits. Aust Endod J 2008;34:52-67. 
[15] Dvir T, Timko BP, Kohane DS, Langer R. Nanotechnological strategies for engineering complex tissues. Nat Nano 2011;6:13-22.

[16] Young CS, Terada S, Vacanti JP, Honda M, Bartlett JD, Yelick PC. Tissue engineering of complex tooth structures on biodegradable polymer scaffolds. Journal of Dental Research 2002;81:695-700.

[17] Wang J, Liu X, Jin X, Ma H, Hu J, Ni L, et al. The odontogenic differentiation of human dental pulp stem cells on nanofibrous poly(L-lactic acid) scaffolds in vitro and in vivo. Acta Biomater 2010;6:3856-63.

[18] Kuang R, Zhang Z, Jin X, Hu J, Gupte MJ, Ni L, et al. Nanofibrous Spongy Microspheres Enhance Odontogenic Differentiation of Human Dental Pulp Stem Cells. Advanced Healthcare Materials 2015;4:1993-2000.

[19] Madden LR, Mortisen DJ, Sussman EM, Dupras SK, Fugate JA, Cuy JL, et al. Proangiogenic scaffolds as functional templates for cardiac tissue engineering. Proceedings of the National Academy of Sciences 2010;107:15211-6.

[20] Madihally SV, Matthew HWT. Porous chitosan scaffolds for tissue engineering. Biomaterials 1999;20:1133-42.

[21] Blaker JJ, Maquet V, Jérôme R, Boccaccini AR, Nazhat SN. Mechanical properties of highly porous PDLLA/Bioglass ${ }^{\circledR}$ composite foams as scaffolds for bone tissue engineering. Acta Biomaterialia 2005;1:643-52.

[22] Liu X, Jin X, Ma PX. Nanofibrous hollow microspheres self-assembled from star-shaped polymers as injectable cell carriers for knee repair. Nature Materials 2011;10:398-406.

[23] Chen F, Liu C, Mao Y. Bismuth-doped injectable calcium phosphate cement with improved radiopacity and potent antimicrobial activity for root canal filling. Acta Biomaterialia 2010;6:3199-207.

[24] Jain RK. Molecular regulation of vessel maturation. Nature Medicine 2003;9:685-93.

[25] Matsumoto K, Yoshitomi H, Rossant J, Zaret KS. Liver Organogenesis Promoted by Endothelial Cells Prior to Vascular Function. Science 2001;294:559-63.

[26] LeCouter J, Moritz DR, Li B, Phillips GL, Liang XH, Gerber H-P, et al. AngiogenesisIndependent Endothelial Protection of Liver: Role of VEGFR-1. Science 2003;299:890-3.

[27] Griffith LG, Swartz MA. Capturing complex 3D tissue physiology in vitro. Nat Rev Mol Cell Biol 2006;7:211-24.

[28] Yu CY, Boyd NM, Cringle SJ, Alder VA, Yu DY. Oxygen distribution and consumption in rat lower incisor pulp. Arch Oral Biol 2002;47:529-36.

[29] Semenza GL. Hypoxia-inducible factor 1: master regulator of O2 homeostasis. Curr Opin Genet Dev 1998;8:588-94.

[30] Sharp FR, Bernaudin M. HIF1 and oxygen sensing in the brain. Nat Rev Neurosci 2004;5:43748.

[31] Pugh CW, Ratcliffe PJ. Regulation of angiogenesis by hypoxia: Role of the HIF system. Nature Medicine 2003;9:677-84.

[32] Forsythe JA, Jiang BH, lyer NV, Agani F, Leung SW, Koos RD, et al. Activation of vascular endothelial growth factor gene transcription by hypoxia-inducible factor 1 . Mol Cell Biol 1996;16:4604-13.

[33] Manalo DJ, Rowan A, Lavoie T, Natarajan L, Kelly BD, Ye SQ, et al. Transcriptional regulation of vascular endothelial cell responses to hypoxia by HIF-1. Blood 2005;105:659-69.

[34] Yamakawa M, Liu LX, Date T, Belanger AJ, Vincent KA, Akita GY, et al. Hypoxia-inducible factor-1 mediates activation of cultured vascular endothelial cells by inducing multiple angiogenic factors. Circ Res 2003;93:664-73. 
[35] Ahluwalia A, Tarnawski AS. Critical role of hypoxia sensor - HIF-1 $\alpha$ in VEGF gene activation. Implications for angiogenesis and tissue injury healing. Current Medicinal Chemistry 2012;19:90-

7.

[36] Aranha AM, Zhang Z, Neiva KG, Costa CA, Hebling J, Nor JE. Hypoxia enhances the angiogenic potential of human dental pulp cells. J Endod 2010;36:1633-7.

[37] Zhang Z, Hu J, Ma PX. Cell carriers with micropores and nanofibers self-assembled in emulsion for tissue regeneration. In submission.

[38] Wang J, Ma H, Jin X, Hu J, Liu X, Ni L, et al. The effect of scaffold architecture on odontogenic differentiation of human dental pulp stem cells. Biomaterials 2011;32:7822-30.

[39] Ferrara N, Davis-Smyth T. The biology of vascular endothelial growth factor. Endocr Rev 1997;18:4-25.

[40] Shweiki D, Itin A, Soffer D, Keshet E. Vascular endothelial growth factor induced by hypoxia may mediate hypoxia-initiated angiogenesis. Nature 1992;359:843-5.

[41] Sheta EA, Trout H, Gildea JJ, Harding MA, Theodorescu D. Cell density mediated pericellular hypoxia leads to induction of HIF-1 $\alpha$ via nitric oxide and Ras/MAP kinase mediated signaling pathways. Oncogene 2001;20:7624-34.

\section{Figure Captions}

Figure 1. . Nanofibrous spongy microspheres (NF-SMS), nanofibrous microspheres (NF-MS) and solid microspheres (S-MS). a) SEM images of nanofibrous spongy microspheres (NF-SMS, left), nanofibrous microspheres (NF-MS, middle) and smooth microspheres (S-MS, right) with a diameter ranging from 30 to $60 \mu \mathrm{m}$ (Scale bar: $20 \mu \mathrm{m}$ ). b) SEM images of hDPSCs seeded on three types of microspheres for 24 hours. Left: hDPSCs on NF-SMS. The attachment of cells was on both the surface and interior of the spheres, with abundant cellular extensions. Middle: hDPSCs on NF-MS. The attachment of cells was shown only on the surface of NF-MS with fewer cellular processes than NFSMS. Right: hDPSCs spread out on S-MS surface with limited cellular processes (Scale bar: $10 \mu \mathrm{m})$.

\section{Figure 2. Nanofibrous spongy microspheres (NF-SMS) provided a beneficial} microenvironment for the proliferation of human dental pulp stem cells (hDPSCs) and the formation of blood vessels. a) Growth curve of hDPSCs cultured on NF-SMS, NF-MS and S-MS, measured by quantifying the DNA content at various time points $(\mathrm{n}=$ 3). $\Delta: \mathrm{p}<0.05$ NF-SMS vs NF-MS, \#: p < 0.05 NF-SMS vs S-MS, *: p < 0.05 NF-MS vs S-MS. b) Subcutaneous injection of hDPSCs/microsphere complexes into nude mice for 
4 weeks. Solid black triangles indicate microspheres; hollow triangles indicate blood vessels (Scale bar: $50 \mu \mathrm{m}$ ). Left: NF-SMS group. NF-SMS promoted abundant connective tissue growth and rich blood vessel formation. Most of the NF-SMS were degraded. Middle: NF-MS group. NF-MS promoted cell growth with less blood vessel formation than NF-SMS. A certain amount of NF-MS still persisted within the neo tissue. Right: S-MS group. S-MS significantly limited connective tissue growth and blood vessels formation. Most of S-MS remained within the neo tissue.

\section{Figure 3. VEGF expression of hDPSCs on NF-SMS was enhanced in hypoxic culture} than in normoxic culture. a) A schematic illustration of the self-made bioreactor for the hDPSCs/NF-SMS suspension culture under hypoxic or normoxic conditions, where the suspension was stirred gently at $37^{\circ} \mathrm{C}$. To create a hypoxic environment, the bioreactor was vacuumed and purged with a mixture of gases comprised of $2 \% \mathrm{O}_{2}, 5 \% \mathrm{CO}_{2}$ and 93 $\mathrm{N}_{2}$ for three times. In normoxic culture, $21 \% \mathrm{O}_{2}, 5 \% \mathrm{CO}_{2}$ and $74 \% \mathrm{~N}_{2}$ gas mixture was used instead. b) Immunofluorescence staining was used to examine the HIF-1 $\alpha$ expression of hDPSCs cultured on NF-SMS under normoxic or hypoxic conditions at 1,3, 7 and 10 days. Blue: nuclei; green: HIF-1 $\alpha$; red: F-actin. Positive staining of HIF-1 $\alpha$ was seen in the hypoxia group at every time point $(1,3,7$ and 10 days). Scale bar: $10 \mu \mathrm{m}$. c) Immunofluorescence staining was used to examine the VEGF expression of hDPSCs on NF-SMS under normoxic or hypoxic conditions at 1, 3, 7 and 10 days. Blue: nuclei; Green: VEGF; Red: F-actin. Positive VEGF staining was observed in the hypoxia group at 3,7 and 10 days. Scale bar: $10 \mu \mathrm{m}$.

Figure 4. Hypoxia induced VEGF gene expression of hDPSCs on NF-SMS. a) Realtime PCR analysis indicated that VEGF mRNA expression level of hDPSCs on S-MS in the hypoxia group was not different from that in the normoxia group at day 1 and day 3 , even became lower than in the normaxia group at day 7 and day $10 . * * \mathrm{p}<0.05$. b) Realtime PCR analysis indicated that VEGF mRNA expression level of hDPSCs on NF-SMS was significant higher in the hypoxia group than in the normoxia group at day 3 and day 7. $* * \mathrm{p}<0.05$. 
Figure 5. Hypoxia primed DPSCs/NF-SMS complexes promoted angiogenesis.

a) H\&E staining (Left panel) and CD31 staining (Right panel) of tissues harvested 4 weeks after subcutaneous injection of hypoxia-/normoxia-primed hDPSCs/NF-SMS into nude mice. H\&E staining (Left panel) showed abundant tissue formation in both groups. CD31 staining (Right panel) showed more microvessels in the hypoxia group (Bottom) than in the normoxia group (Top). Scale bars: $100 \mu \mathrm{m}$. b) The numbers of blood vessels were quantified, and the hypoxia group had significantly more blood vessels than normoxia group $(\mathrm{n}=3)$. $* \mathrm{p}<0.05$.

Figure 6. Dental pulp tissue regeneration promoted by hypoxia-primed hDPSCs/NFSMS in a subcutaneous tooth implantation model. Human DPSCs cultured on NFSMS were primed under hypoxic or normoxic conditions for 3 days, and were injected into the cavity of evacuated rabbit molars, which were then subcutaneously implanted into nude mice for 4 weeks. a) Grossly, the harvested rabbit molars from the hypoxia group had a redder appearance than the three control groups. b) The number of blood vessels were quantified, and the hypoxia group had significantly more blood vessels than the other 3 groups $(\mathrm{n}=3) . * * \mathrm{p}<0.05$. c) Histologic analysis of the harvested rabbit molars. H\&E staining (reconstructed from multiple microscopic images) showed that there were more neo tissues in the hypoxia group than in the three control groups. Odontoblast-like cells were along the dentin pulp interface in hypoxia group verified by DSPP IHC staining. Weak DSPP staining in the dentin-pulp interface was shown in the normoxia group. Negative DSPP staining was shown in both NF-SMS alone group and hDPSCs alone group. CD31 IHC staining showed that there were more microvessels in the hypoxia group than in the normoxia group. Fewer and smaller blood vessels were observed in hDPSCs alone group and NF-SMS alone group. Scale bars: $50 \mu \mathrm{m}$.

\section{Figure 7. Pulp tissue regeneration enhanced by hypoxia primed hDPSCs/NF-SMS in} maxillary first molar of nude rats. From left to right, the first column is the normal pulp, the second column is the unfilled pulp canal group, the third column is the normoxia group, and the last column is the hypoxia group. $\mathrm{H} \& \mathrm{E}$ staining showed that no pulp-like tissue was formed in unfilled group while neo pulp-like tissue formed in 
normoxia and hypoxia groups. DSPP IHC staining was positive in the hypoxia group and the normal pulp group at the dentin-pulp interface (the triangle pointed to the dentin-pulp interface). CD31 staining showed more blood vessels in the hypoxia group than in the normoxia group (marked with arrows). 


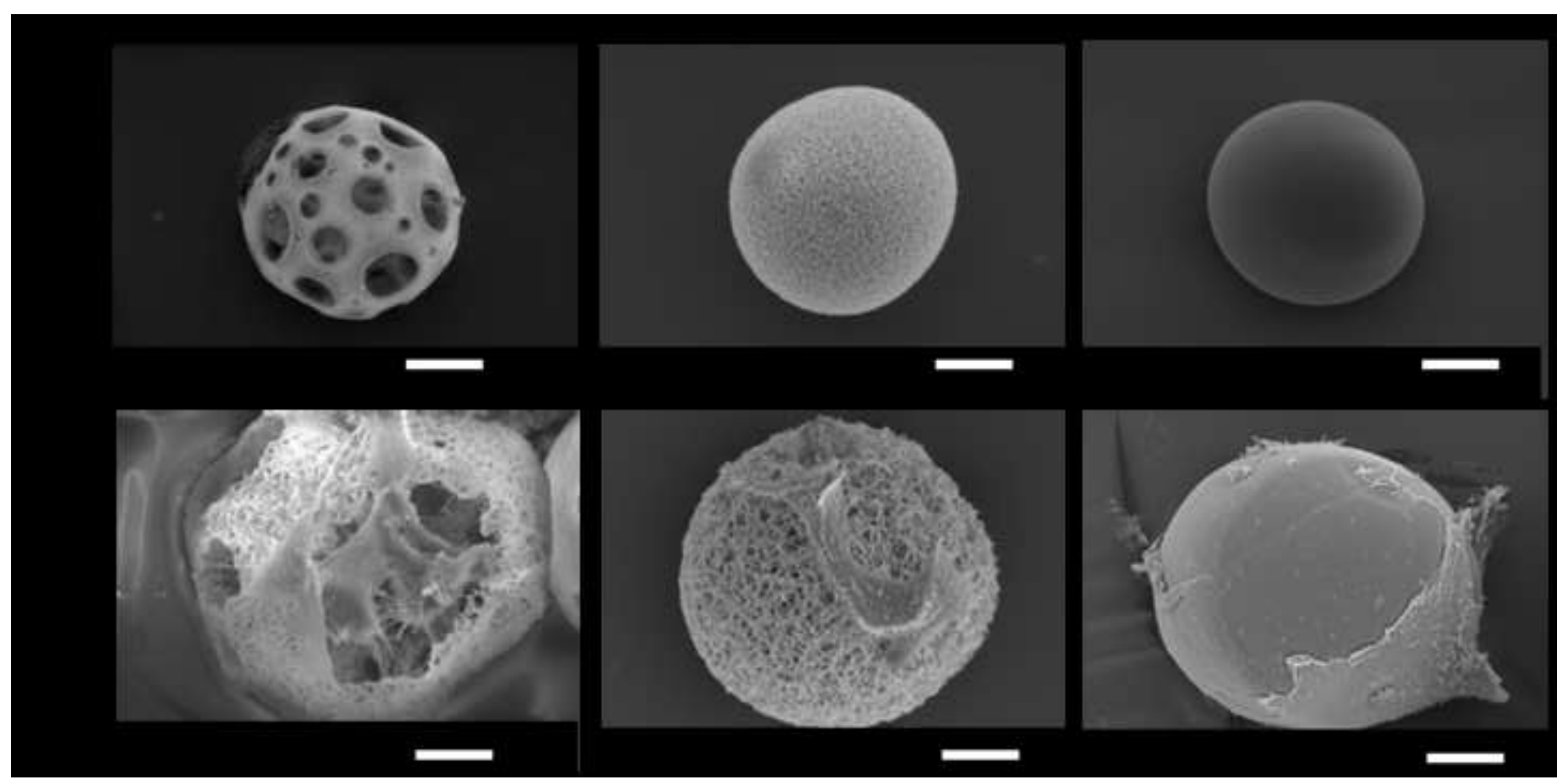




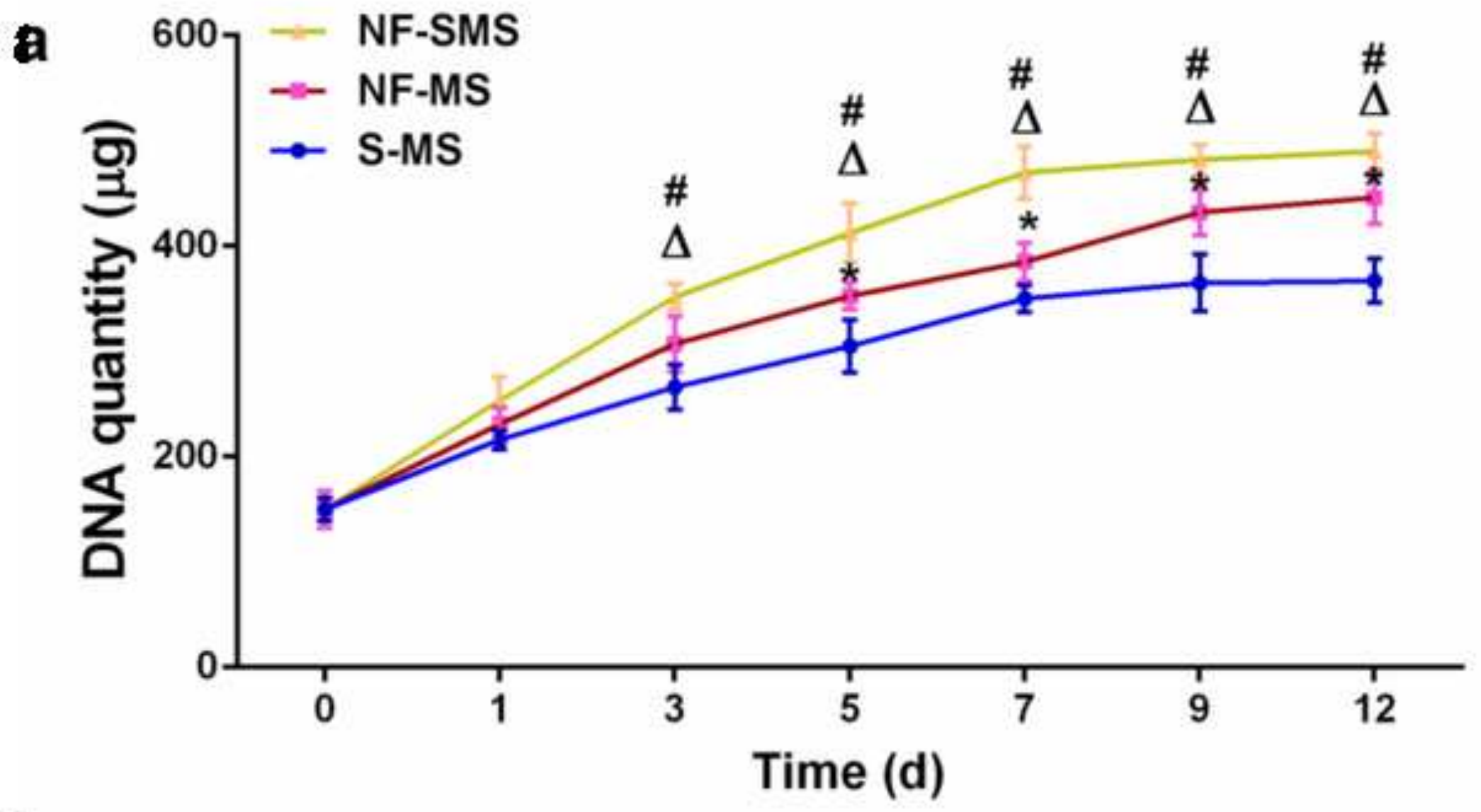

b

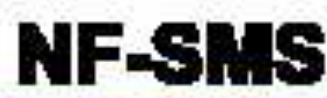

NFAMS

S-MS

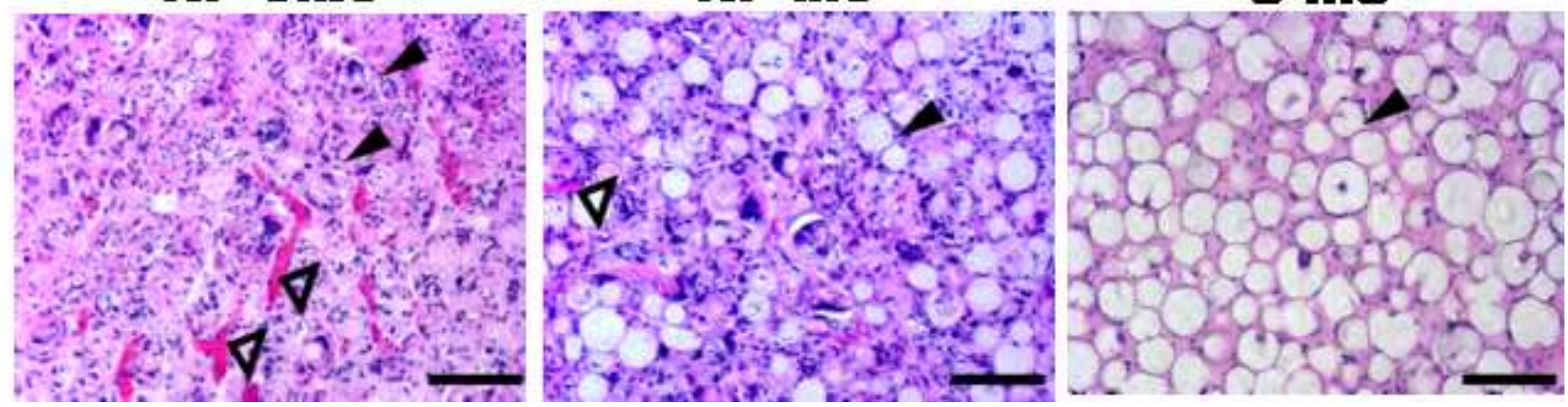


a) Hyode Don wactor
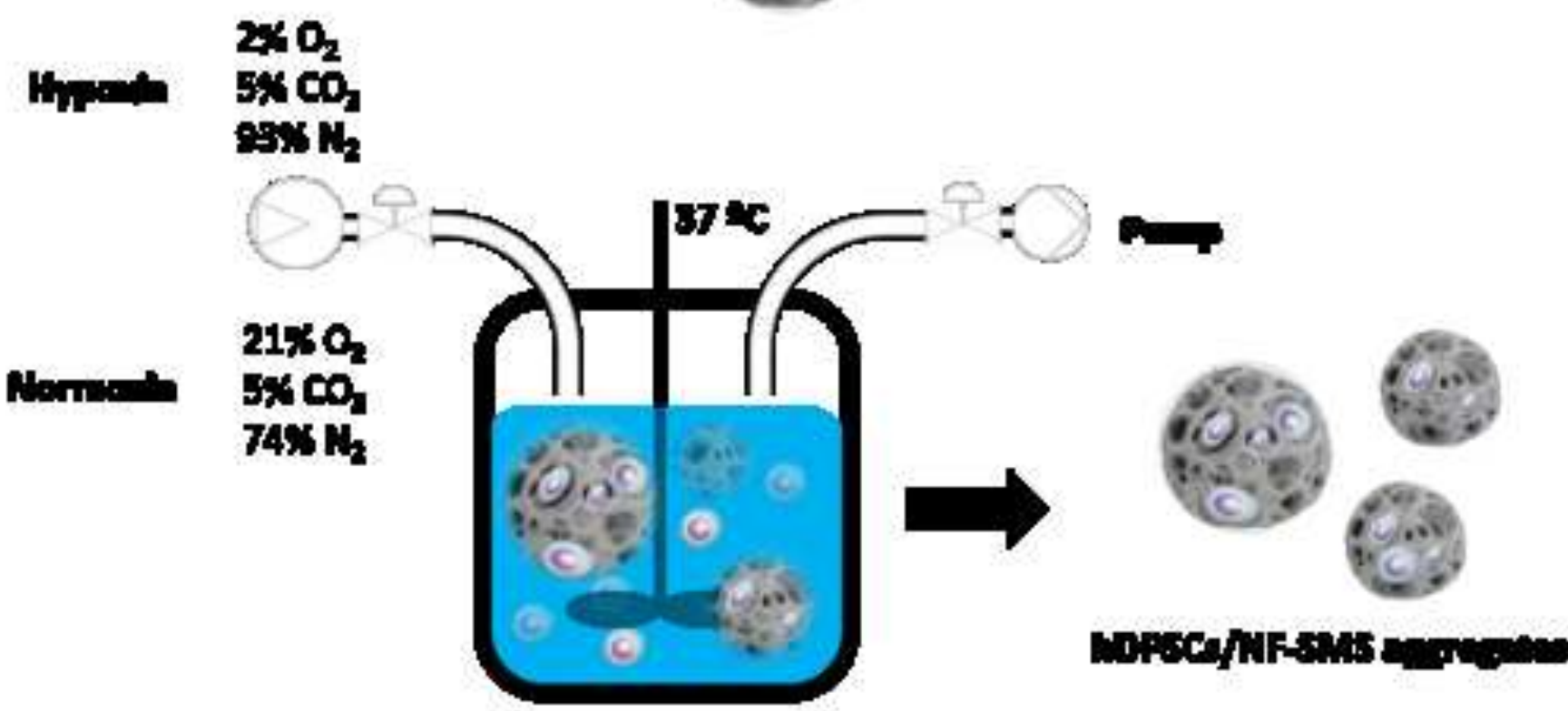

b) Hif-1esthing
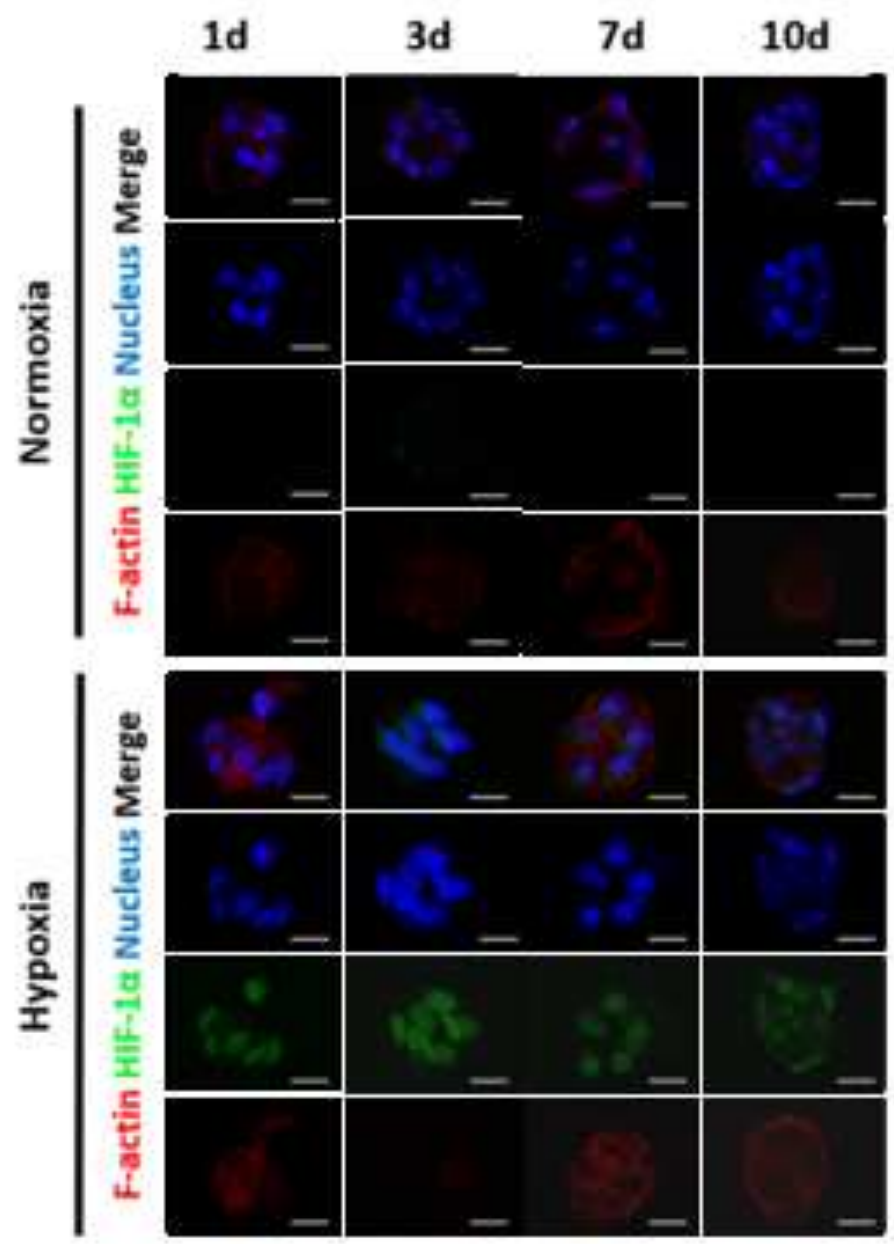

c) Verosth
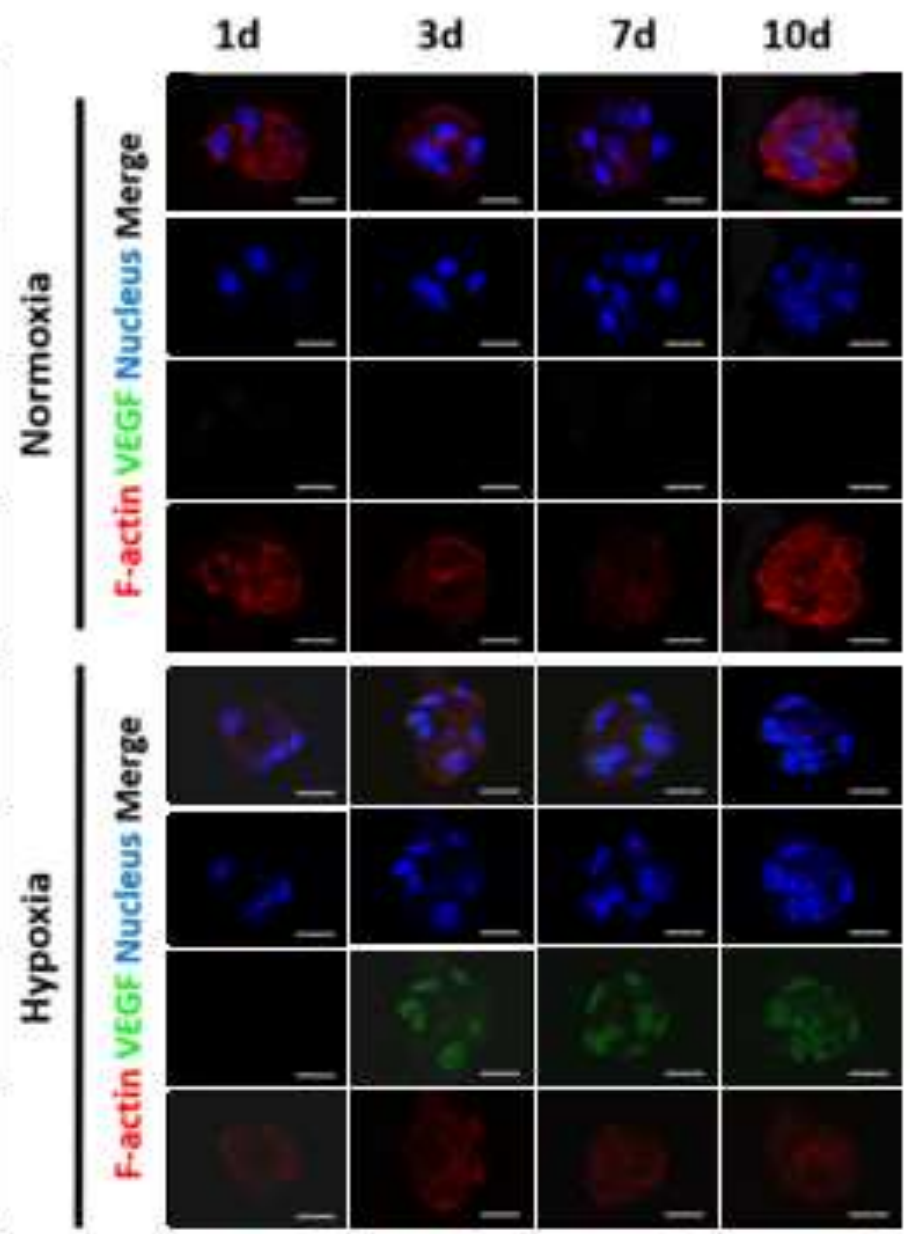
a)

$\square$ Normoxia

- Hypoxia

hDPSCs on S-MS

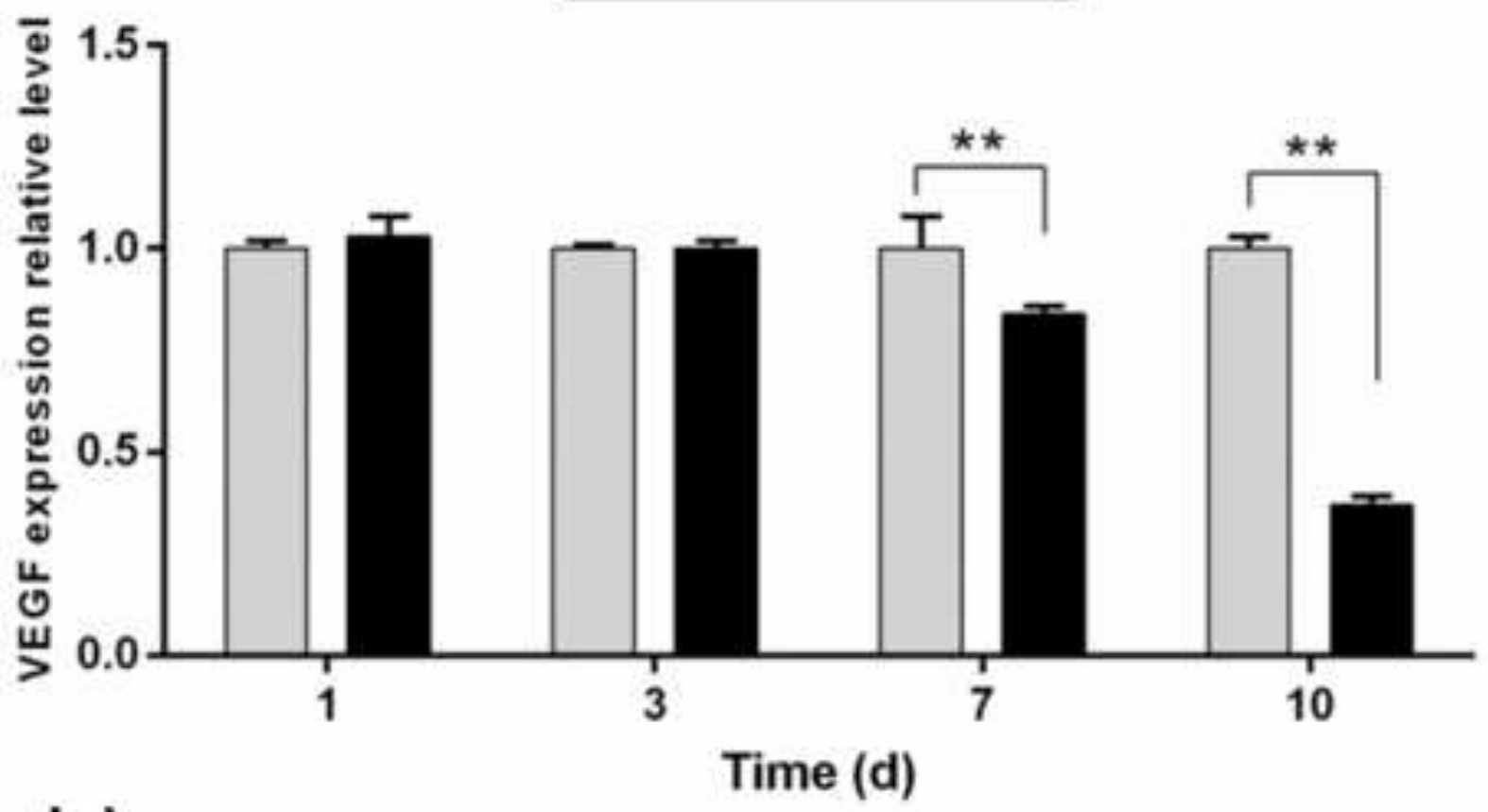

b)

\section{hDPSCs on NF-SMS}

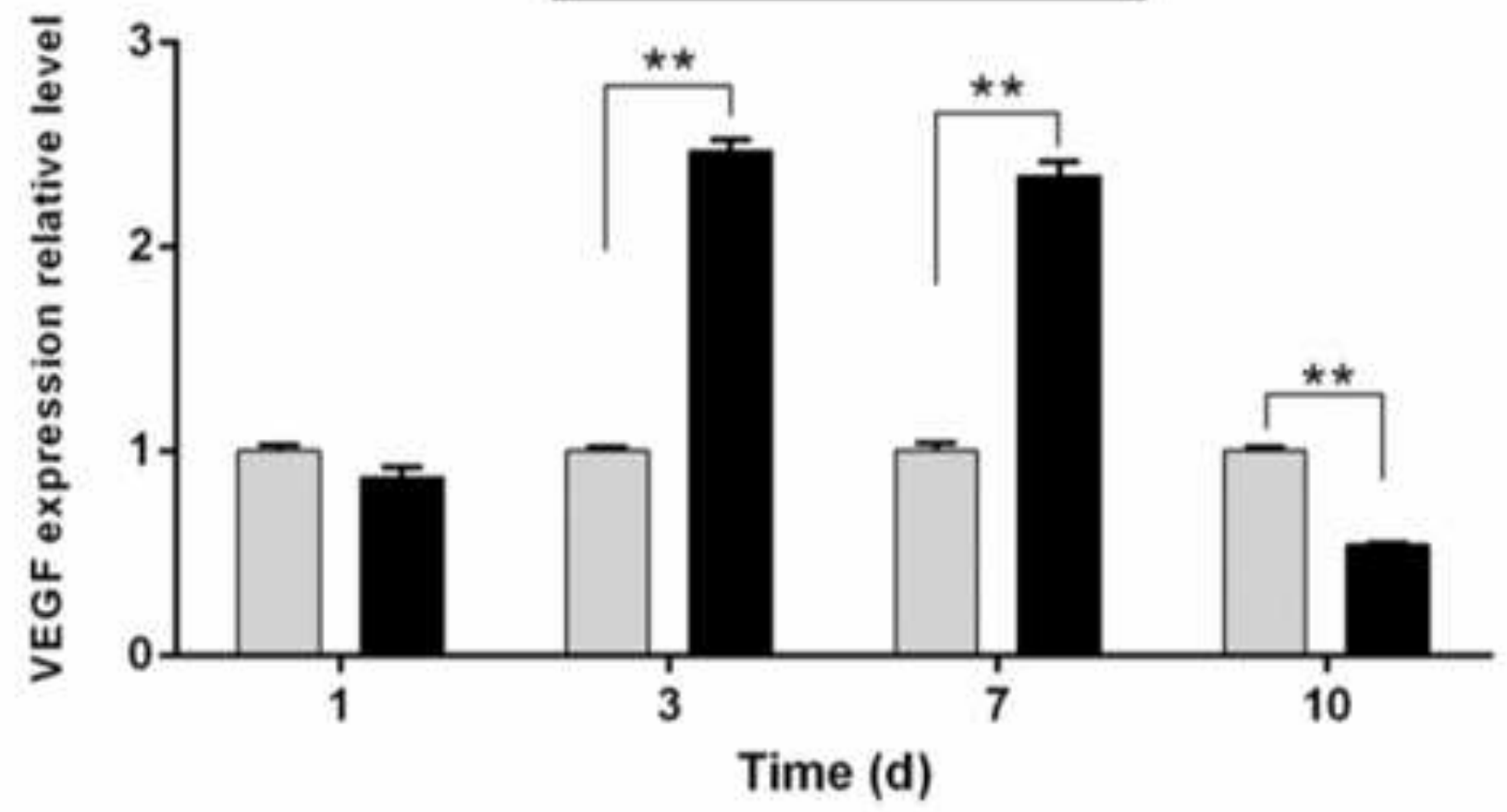


a Subcutaneous Injection H \& $E$

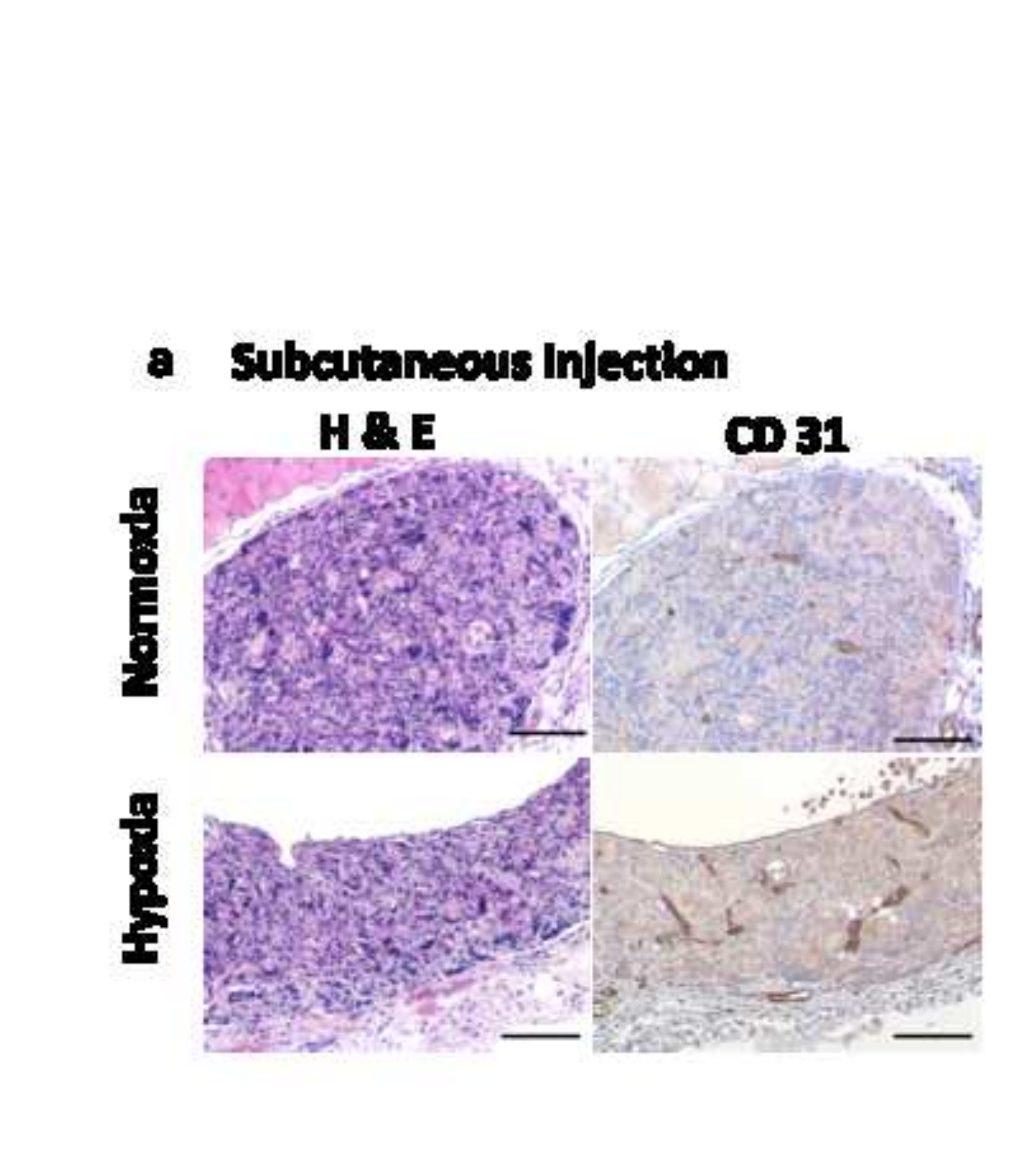

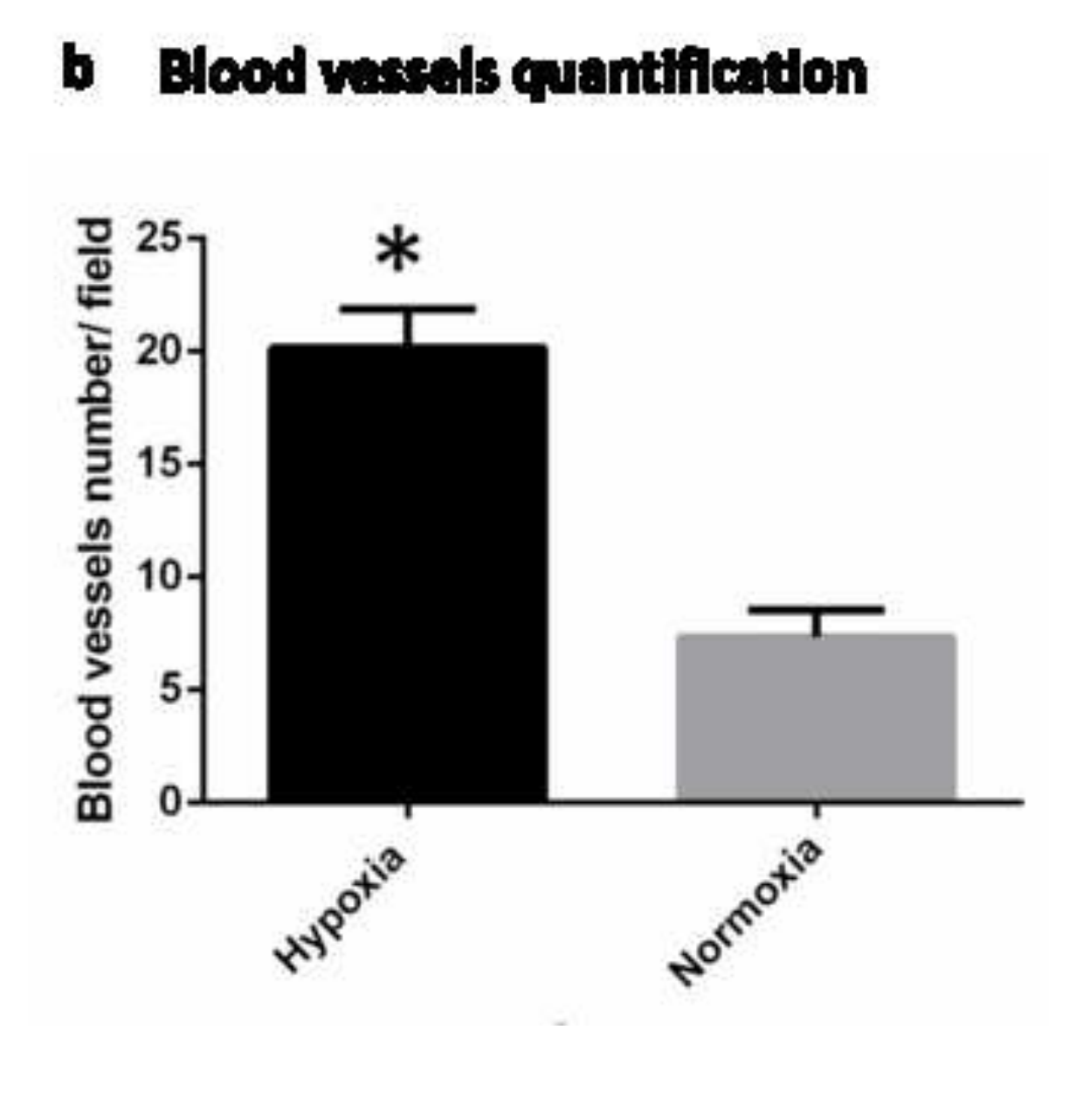

\section{b Blood vescels quantification}

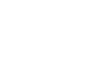
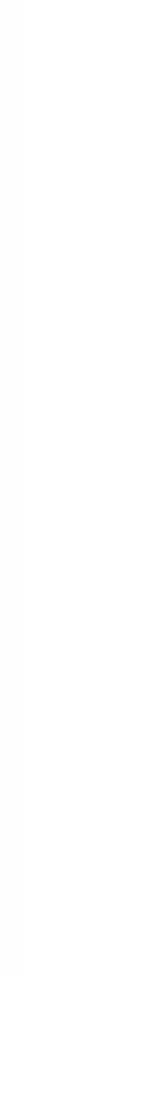

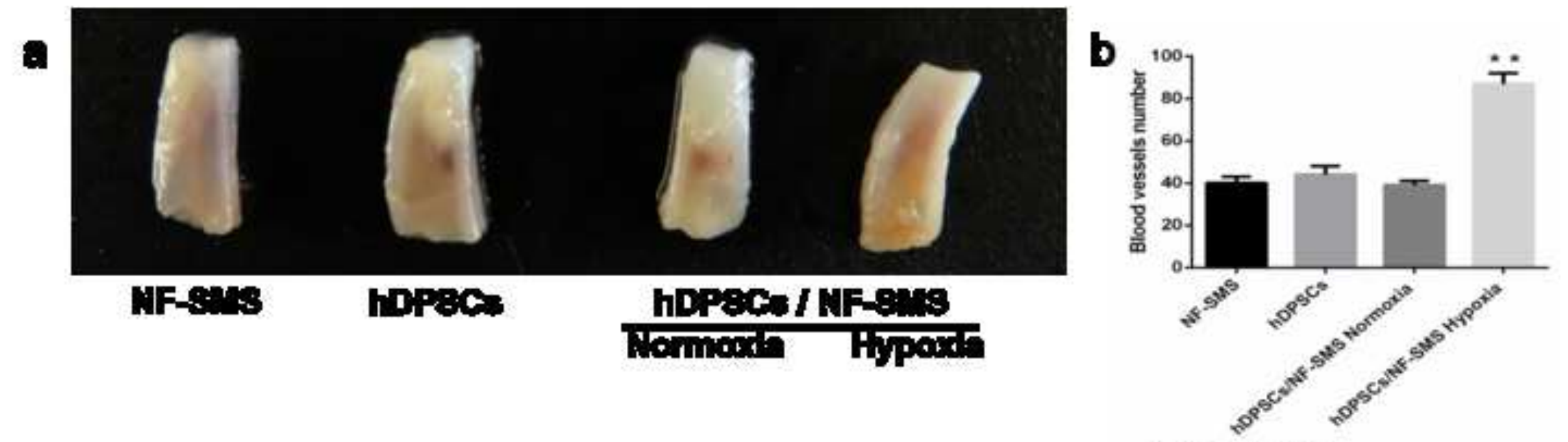

6
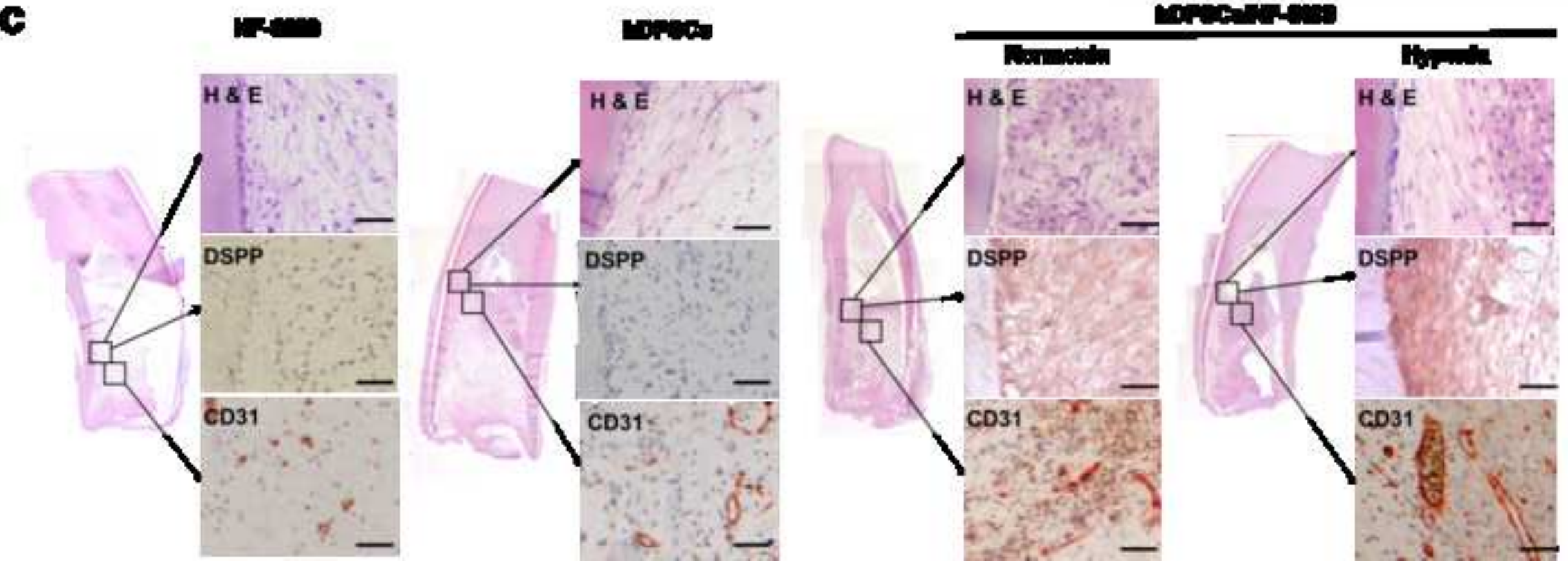
